\title{
Extracto de Macleaya cordata como uso alternativo a la bacitracina en lechones
}

\section{Macleaya cordata extract for use alternative to bacitracin in piglets}

\author{
Manuel Paredes ${ }^{1^{*}}$ (D), Víctor Paico ${ }^{1}$ (D) José Mantilla $^{1}$ (D) Arif Mustafa $^{2}$ (D)
}

\section{RESUMEN}

Veintisiete lechones Yorkshire $\mathrm{x}$ Duroc de veintiocho días de edad fueron alimentados con una de las tres dietas experimentales que consistían en una dieta basal (DB), la dieta basal suplementada con $50 \mathrm{mg}$ de extracto de Macleaya cordata por kg de dieta (DB-EMC) y la dieta basal suplementada con $50 \mathrm{~g}$ de bacitracina de zinc por kg de dieta (DB-B) durante un período de cinco semanas. En comparación de los animales que recibieron las dietas suplementadas con extracto de Macleaya cordata (EMC) y bacitracina, los cerdos alimentados con la dieta basal mostraron una menor ganancia media diaria (GMD) y una conversión alimenticia más pobre. En general, el rendimiento del crecimiento y el peso corporal final en los lechones DB-EMC y DB-B fueron mejores que el de los lechones DB $(p<0,05)$. En las semanas 1,2 y 5 , la GMD fue mayor en comparación con $\mathrm{DB}(\mathrm{p}<0,05)$. La incidencia de diarreas cambió gradualmente de la semana 1 ( 5 en DB-EMC, 4 en DB-B y 6 en DB) a la semana 2 (1 en DB-EMC, 1 en DB-B y 5 en DB). En general, los tratamientos aditivos DB-EMC y DB-B tuvieron una disminución en la incidencia de diarreas en comparación con el control negativo. En conclusión, la sustitución de bacitracina por sanguinarina del EMC en lechones destetados es una buena alternativa con rendimientos satisfactorios.

Palabras claves: sanguinarina, bacitracina, lechón, crecimiento.

\begin{abstract}
Twenty-seven Yorkshire $x$ Duroc piglets age of 28 days were fed one of trhee experimental diets consisting of a basal $\operatorname{diet}(\mathrm{DB})$, the basal diet supplemented with $50 \mathrm{mg}$ Macleaya cordata extract per kg diet (DB-EMC) and the basal diet supplemented with $50 \mathrm{~g}$ zinc bacitracine per $\mathrm{kg}$ diet (DB-B) over a period of five weeks. Compared to the animals receiving the Macleaya cordata extract EMC and bacitracin supplemented diets, pigs fed the basal diet showed a decrease in daily gain and poorer feed conversion. In overall growt performance and final body weight in the piglets DB-EMC and DB-B was larger than that in the DB ( $<<0.05)$. At weeks 1,2 and 5 the daily average gain (GMD) was higher compared to DB $(\mathrm{p}<0.05)$. The diarrheas incidence shifted gradually from week 1 (5 DB-EMC, 4 DB-B and 8 $\mathrm{DB})$ to week 2 (5 DB-EMC, 4 DB-B and $6 \mathrm{DB})$. At overall the additive treatments DB-EMC and DB-B gave a decrease in diarrheas incidence compared to negative control. In conclusion, the replacement of bacitracin by sanguinarine from the EMC in weaned piglets is good alternative with satisfactory yields.
\end{abstract}

Keywords: sanguinarine, bacitracin, piglet, growth.

\footnotetext{
Universidad Nacional de Cajamarca, Facultad de Ingeniería en Ciencias Pecuarias, Cajamarca, Perú.

${ }^{2}$ McGill University, Department of Animal Science, Montreal, Canada.

Autor de Correspondencia.E-mail:mparedes@unc.edu.pe
} 


\section{INTRODUCCIÓN}

Lechón es la denominación que se da al cerdo desde su nacimiento, durante la lactancia y luego del destete, hasta los setenta días de edad (Cadillo, 2008). El lechón es importante en la granja porcina porque, parte de ellos se incorporan al plantel de reproducción, otros ingresan al proceso de engorde y también algunos lechones se constituyen en un producto terminado, por ser la carne de lechón un alimento muy apreciado en el sector gastronómico. Cuando el lechón se destina para consumo humano, no debería ingerir antibióticos a fin de evitar contenidos residuales en la carne y la emergencia de patógenos resistentes a antibióticos, que afecta la producción alimentaria (Casana, 2017) y la salud pública; debiendo intensificarse la evaluación de nuevos aditivos alimenticios que sustituyan a los antibióticos promotores de crecimiento (APC) del pienso de los lechones destetados.

La dieta es uno de los principales reguladores de la microbiota intestinal de los cerdos (Waititu et al., 2017). Muchas estrategias de manejo alimenticio han sido evaluadas y utilizadas en la industria porcina en un intento por mitigar los efectos negativos del estrés del destete en el rendimiento y la salud de los lechones (Nagai y Buttrey, 2018). El lechón destetado y separado de su madre pasa a consumir una dieta rica en lípidos y polisacáridos provenientes de maíz y soya principalmente, teniendo que hacer frente al estrés inflamatorio inducido por los lipopolisacáridos, que provocan una producción alterada de citocinas y quimiocinas inflamatorias (Tucureanu et al., 2018). Los lechones después del destete tienen un intestino inmaduro y órganos inmunes que no han completado su desarro1lo, siendo muy vulnerables a los microorganismos invasores (Lauridsen, 2020). El estrés al destete disminuye la capacidad digestiva y de absorción del intestino delgado (Wang et al., 2019), induciendo cambios estructurales y funcionales en el intestino delgado, pero también en la morfología intestinal, fisiología y salud del ciego (Deng et al., 2020), debido a que, durante el período de destete, la fermentación en el íleon, ciego y colon proximal pasa de una fermenta- ción proteolítica a una de carbohidratos (Pellikaan et al., 2010).

En el Perú todavía se utilizan una serie de fármacos en la dieta del lechón para contrarrestar el proceso inflamatorio; sin embargo, existe la preocupación mundial desde hace varias décadas, para sustituir los APC como la bacitracina, en alimentación animal ( $\mathrm{Li}$ et al., 2020). Habiéndose encontrado una mejor capacidad de los lechones alimentados con OH-metionina a través de suplementos maternos y durante el período posterior al destete para hacer frente al estrés inflamatorio inducido por lipopolisacáridos (Xu et al., 2017). Por otro lado, los fitobióticos, definidos como productos derivados de especies vegetales se pueden agregar al alimento como alternativas a los APC (Mohammadi Gheisar y Kim, 2018). Macleaya cordata, es una especie vegetal de la familia Papaveraceae, que viene siendo utilizada como aditivo en alimentación de cerdos (Kosina et al., 2004). Macleaya cordata contiene alcaloides importantes, que incluyen protopina y sus metabolitos como la benzofenantridina o sanguinarina (Chen et al., 2009). Los alcaloides benzofenantridínicos son biosintetizados de las protopinas por acción de la enzima protopina 6-hidroxilasa, que da lugar a la formación de 6-hidroxiprotopina que espontáneamente sufre transposición hacia dihidrosanguinarina, con un átomo de nitrógeno proveniente, posiblemente del aminoácido triptófano (Díaz, 2003); aunque, luego se determinó que la sanguinarina no tiene ningún efecto beneficioso sobre la disponibilidad o metabolismo del triptófano (Blank et al., 2010).

Se ha determinado que la suplementación con sanguinarina facilita el establecimiento de bacterias beneficiosas en el tracto gastrointestinal de los cerdos, inhibe la colonización de bacterias patógenas (Newton et al., 2001) y disminuye el producto final de la fermentación en el ciego y el colon proximal durante los primeros días después del destete (Pellikaan et al., 2010). También se ha comprobado que la sanguinarina componenete del producto comercial Sangrovit ${ }^{\circledR}$ tiene características antimicrobianas y antiinflamatorias, a nivel de granja en condiciones higiénicas críticas (Blank et al., 
2010). Del mismo modo se ha determinado que el extracto de Macleaya cordata (EMC) mejoró el crecimiento (Kantas et al., 2015) y disminuyó la incidencia de diarreas en cerdos (Ni et al., 2016). Se determinó en cerdos de 35 días de edad suplementados con $50 \mathrm{mg}$ de $\mathrm{MCE} / \mathrm{kg}$ de dieta, durante 28 días, mayor altura de la vellosidad intestinal (AV) y mayor relación con la profundidad de cripta (PC) en el íleon, con una tendencia a disminuir la relación $\mathrm{AV} / \mathrm{PC}$ en el duodeno, y con disminución considerable de bacterias del género Lactobacillus en el duodeno, yeyuno e íleon (Li et al., 2020).

El presente estudio tuvo por objetivo evaluar la sustitución del APC bacitracina por el fitobiótico extracto de Macleaya cordata en la dieta de los lechones cruzados durante cinco semanas post destete, considerando los efectos sobre los indicadores de crecimiento e incidencia de diarreas.

\section{MATERIALES Y MÉTODOS}

\section{Animales y alojamiento}

El experimento se llevó a cabo en la Granja Huacariz, ubicada en la cabecera del valle interandino de Cajamarca, a 2785 m.s.n.m. Los lechones para el presente estudio se obtuvieron de cerdas de segundo parto. Luego de tres días de acostumbramiento a la dieta basal, veintisiete lechones destetados, cruzados Yorkshire x Duroc de ambos sexos con una edad de 28 días ( \pm 3 días) y un peso corporal de $8,7 \pm 2,3 \mathrm{~kg}$ (media \pm DE) en el momento del inicio del experimento (día 0), fueron bloqueados en tres grupos según peso corporal (pequeño, mediano y grande), y asignado al azar a uno de los tres tratamientos dietéticos. Los lechones fueron alojados en grupo de tres animales por corral, dos machos y una hembra. Cada corral tuvo una superficie de $4 \mathrm{~m}^{2}$, con cama de viruta, equipados con comedero automático y bebedero tipo chupón, con temperatura controlada de $22 \pm 1^{\circ} \mathrm{C}$ durante la primera semana experimental.

\section{Tratamientos dietéticos}

Los tres tratamientos dietéticos consistieron en una dieta basal (DB), dieta basal suplementada con $50 \mathrm{mg}$ de EMC por kg de dieta (DB-EMC) y la dieta basal suplementada con $50 \mathrm{~g}$ de bacitracina por $\mathrm{kg}$ de dieta (DB-B). El EMC, se lo adquirió como Sangrovit ${ }^{\circledR}$, procedente de la empresa Phytobiotics, y contiene $1,75 \%$ de sanguinarina por $\mathrm{kg}$. La Bacitracina se adquirió de distribuidora Montana SA, como Baczin ${ }^{\circledR}$, y contiene $10 \%$ de zinc bacitracina. La dieta basal (Tabla 1) fue formulada para cumplir o exceder los nutrientes y la energía para lechones destetados, recomendados por la NRC (1998). Durante todo el experimento, los cerdos tuvieron acceso ad-libitum a sus dietas experimentales y al agua. El excedente diario de alimento se recogió para obtener la ingesta de alimento.

Tabla 1. Ingredientes y contenido nutricional de la dieta basal utilizada en el experimento

\begin{tabular}{|c|c|}
\hline Ingredientes & $\mathbf{g} / \mathbf{k g}$ \\
\hline Maíz amarillo & 655,0 \\
\hline Torta de soya & 218,0 \\
\hline Harina de pescado & 35,0 \\
\hline Afrecho de trigo & 67,0 \\
\hline Carbonato de calcio & 11,5 \\
\hline Fosfato monocálcico & 5,5 \\
\hline $\mathrm{NaCl}$ & 3,0 \\
\hline Premezcla vitaminas y minerales ${ }^{\text {a }}$ & 5,0 \\
\hline \multicolumn{2}{|c|}{ Contenido nutricional calculado } \\
\hline Materia seca & 892,0 \\
\hline Energía metabolizable, Kcal/kg & 3230,0 \\
\hline Proteína cruda & 185,6 \\
\hline Fibra cruda & 44,1 \\
\hline Arginina & 4,6 \\
\hline Lisina & 11,5 \\
\hline Metionina & 3,7 \\
\hline Treonina & 7,9 \\
\hline Triptófano & 2,0 \\
\hline Calcio & 8,0 \\
\hline Pdisponible & 3,6 \\
\hline
\end{tabular}

${ }^{a}$ Cada kg de premezcla provee: vitamina $\mathrm{A}$ : 10,000 UI; vitamina $\mathrm{D}_{3}$ : $1200 \mathrm{UI}$; vitamina $\mathrm{E}$ : $85 \mathrm{mg}$; vitamina $\mathrm{K}: 4 \mathrm{mg}$; vitamina $\mathrm{B}_{1}: 3 \mathrm{mg}$, vitamina $B_{2}: 6 \mathrm{mg}$; vitamina $B_{6}: 4 \mathrm{mg}$; vitamina $B_{12}: 0.05 \mathrm{mg}$; ácido nicotínico: $40 \mathrm{mg}$, biotina: $0.25 \mathrm{mg}$; ácido pantoténico: $20 \mathrm{mg}$; calcio: $1.14 \mathrm{~g}$; Se:0.36 mg; Fe: $180 \mathrm{mg}, \mathrm{Cu}: 165 \mathrm{mg}$; Mn: $50 \mathrm{mg}$, Co: $0.1 \mathrm{mg} ; \mathrm{I}: 2 \mathrm{mg}$, and Phytase FTU 500.

\section{Determinación de los parámetros de crecimiento}

Semanalmente se sumó la cantidad ingerida de alimento por los cerdos de cada corral (AISC) y luego se determinó la ingesta diaria de alimento por lechón (IDA), mediante la división de AISC por el número de 
lechones por corral y sobre los días de la semana. El peso corporal (PC) de cada animal se controló semanalmente en una balanza electrónica de plataforma (BEP) con capacidad de $30 \mathrm{~kg}$ y precisión de $1 \mathrm{~g}$. En las dos últimas semanas experimentales se utilizó una BEP de $300 \mathrm{~kg}$ de capacidad y 5 g de precisión. La ganancia media diaria (GMD) de cada lechón por semana se determinó por diferencia del PC de las dos últimas semanas, dividido por el número de días de la semana. La relación de la IDA y GMD se usó para calcular el índice de conversión alimenticia (ICA).

\section{Manejo y control sanitario}

Los lechones fueron desparasitados durante la pesada inicial, con ivermectina $2 \%$ a razón de $0.3 \mathrm{ml} /$ animal. A los 35 días de edad, al controlar el peso de los lechones en la primera semana experimental se vacunó contra cólera porcino. Cada caso de diarrea presentado por lechón fue registrado y tratado con Baytril (10\% enrofloxacina) inyectable a razón de $1 \mathrm{ml}$ por lechón.

\section{Análisis estadístico}

El análisis estadístico se realizó utilizando el software estadístico Statistical Analysis System, 2014, mediante el procedimiento MIXED (SAS Institute Inc., Cary, North Carolina, USA). Los datos de los tratamientos fueron sometidos a un análisis de varianza con el modelo lineal general. Las diferencias entre medias de los tratamientos se determinaron mediante prueba de Duncan $(p<0,05)$. Las diferencias entre tratamientos para los casos de diarreas se calcularon con pruebas de chi cuadrado $\mathrm{X}^{2}$.

\section{RESULTADOS Y DISCUSIÓN}

Los parámetros de crecimiento de los lechones PC, GMD, IDA e ICA por grupos experimentales evaluados semanalmente durante todo el período de prueba se muestran en la Tabla 2.

Tabla 2. Promedios de peso corporal inicial (PCI), IDA, GMD e ICA según tratamientos, determinados semanalmente durante el experimento

\begin{tabular}{|c|c|c|c|c|c|}
\hline & DB & DB-EMC & DB-B & SEM & Valor-p \\
\hline \multicolumn{6}{|c|}{ Semana 1} \\
\hline PCI, kg & 8,45 & 8,88 & 8,80 & 0,12 & 0,342 \\
\hline IDA, g/d & 513 & 508 & 504 & 3 & 0.546 \\
\hline GMD, g/d & $270^{\mathrm{b}}$ & $320^{\mathrm{a}}$ & $380^{\mathrm{a}}$ & 53 & 0,047 \\
\hline ICA & $1,95^{\mathrm{a}}$ & $1,72^{\mathrm{ab}}$ & $1,38^{\mathrm{b}}$ & 0,16 & 0,045 \\
\hline \multicolumn{6}{|c|}{ Semana 2} \\
\hline PCI, kg & $10,34^{\mathrm{b}}$ & $11,11^{\mathrm{a}}$ & $11,44^{\mathrm{a}}$ & 0,32 & 0,045 \\
\hline IDA, g/d & 798 & 759 & 796 & 13 & 0.453 \\
\hline GMD, g/d & $353^{\mathrm{b}}$ & $470^{\mathrm{a}}$ & $482^{\mathrm{a}}$ & 42 & 0,041 \\
\hline ICA & $2,38^{a}$ & $1,90^{\mathrm{b}}$ & $1,71^{\mathrm{b}}$ & 0,20 & 0,034 \\
\hline \multicolumn{6}{|c|}{ Semana 3} \\
\hline PCI, kg & $12,81^{\mathrm{b}}$ & $14,40^{\mathrm{a}}$ & $14,82^{\mathrm{a}}$ & 0,61 & 0,034 \\
\hline IDA, g/d & 1104 & 1120 & 1061 & 18 & 0,654 \\
\hline GMD, g/d & 715 & 760 & 653 & 38 & 0,202 \\
\hline ICA & 1,56 & 1,56 & 1,71 & 0,05 & 0,087 \\
\hline \multicolumn{6}{|c|}{ Semana 4} \\
\hline PCI, kg & $17,82^{\mathrm{b}}$ & $19,72^{\mathrm{a}}$ & $19,39^{\mathrm{a}}$ & 0,58 & 0,036 \\
\hline IDA, g/d & 1204 & 1343 & 1232 & 42 & 0,536 \\
\hline GMD, g/d & 643 & 670 & 703 & 38 & 0,102 \\
\hline ICA & $1,87^{\mathrm{b}}$ & $2,02^{\mathrm{a}}$ & $1,76^{\mathrm{b}}$ & 0,07 & 0,045 \\
\hline \multicolumn{6}{|c|}{ Semana 5} \\
\hline PCI, kg & $22,32^{b}$ & $24,41^{\mathrm{a}}$ & $24,31^{\mathrm{a}}$ & 0,68 & 0,032 \\
\hline IDA, g/d & 1480 & 1588 & 1587 & 36 & 0,463 \\
\hline GMD, g/d & $695^{\mathrm{b}}$ & $802^{\mathrm{a}}$ & $767^{\mathrm{a}}$ & 38 & 0,048 \\
\hline ICA & 2,15 & 1,99 & 2,07 & 0,04 & 0,237 \\
\hline
\end{tabular}

a,b Letras diferentes en el superíndice de la misma fila indican diferencias estadísticas $(\mathrm{p}<0,05)$. DB: Dieta basal. DB-EMC: Dieta basal +50 $\mathrm{mg} / \mathrm{kg}$ de extracto de Macleaya cordata. DB-B: Dieta basal $+50 \mathrm{~g} / \mathrm{kg}$ de zinc bacitracina. IDA: Ingesta diaria por animal. GMD: Ganancia media diaria. ICA: Indice de conversión alimenticia.

26 Rev. de investig. agroproducción sustentable 4(2): 23-30, 2020 ISSN: 2520-9760 
Aunque los lechones de todos los tratamientos comenzaron con un similar PC, se mostró un PC significativamente mayor en los grupos DB-EMC y DB-B al final de la etapa de prueba $(p<0,05)$ en comparación con el grupo DB. Se observaron diferencias significativas en la GMD en diferentes semanas. Los tratamientos DB-EMC y DB-B mostraron mayor GMD en las semanas 1, 2 y 5 en comparación con el grupo DB. No se observaron diferencias ente tratamiento en cuanto a la IDA, en ninguna de las comparaciones semanales. Se observaron diferencias significativas en el ICA sólo en dos semanas y entre diferentes grupos, sin tener una visión clara a favor de un tratamiento específico. Los tratamientos DB-EMC y DB-B mostraron mejor ICA que $\mathrm{DB}$ en la semana 2, mientras que los grupos $\mathrm{DB}$ EMC y DB mostraron mejor ICA que DB-B en la semana 4. Sin embargo, los tratamientos con EMC y bacitracina parecen mejorar el ICA en comparación con el control negativo, al final de todo el periodo experimental. En la Tabla 3 se presentan los datos de PC final, IDA, GMD e ICA para el período de prueba total según tratamientos. No se observaron diferencias significativas en incidencia de diarreas entre los grupos experimentales en la primera semana, pero sí en la segunda semana, como se muestra en la Tabla 4. En las siguientes semanas experimentales no se observaron mayores casos de diarreas por tratamientos.

Tabla 3. Promedios de peso corporal final (PCF), IDA, GMD e ICA según tratamientos de todo el período experimental

\begin{tabular}{lccccc}
\hline & DB & DB-EMC & DB-B & SEM & Valor-p \\
\hline PCF, kg & $27,19^{\mathrm{b}}$ & $30,02^{\mathrm{a}}$ & $29,68^{\mathrm{a}}$ & 0,89 & 012 \\
IDA, g/d & 1020 & 1071 & 1028 & 16 & 0,765 \\
GMD, g/d & $535^{\mathrm{b}}$ & $604^{\mathrm{a}}$ & $596^{\mathrm{a}}$ & 0,134 \\
ICA & $1,91^{\mathrm{a}}$ & $1,81^{\mathrm{b}}$ & $1,75^{\mathrm{b}}$ & 0,05 & 0,049 \\
\hline
\end{tabular}

a,b Letras diferentes en el superíndice de la misma fila indican diferencias estadísticas $(\mathrm{p}<0,05)$. DB: Dieta basal. DB-EMC: Dieta basal +50 $\mathrm{mg} / \mathrm{kg}$ de extracto de Macleaya cordata. DB-B: Dieta basal $+50 \mathrm{~g} / \mathrm{kg}$ de zinc bacitracina. IDA: Ingesta diaria por animal. GMD: Ganancia media diaria. ICA: Índice de conversión alimenticia.

Tabla 4. Incidencia de diarreas en lechones destetados luego de la primera y segunda semana, según tratamientos apareados

\begin{tabular}{|c|c|c|}
\hline Tratamientos & $N^{\circ}$ de lechones con diarrea & Significancia estadística \\
\hline \multicolumn{3}{|c|}{ Primera semana } \\
\hline DB vs. DB -EMC & 6 vs. 5 & $\mathrm{~ns}$ \\
\hline DB vs. DB -B & $6 \mathrm{vs} .4$ & ns \\
\hline DB-B vs. DB -EMC & 4 vs. 5 & ns \\
\hline \multicolumn{3}{|c|}{ Segunda semana } \\
\hline DB vs. DB -EMC & 5 vs. 1 & $*$ \\
\hline DB vs. DB -B & 5 vs. 1 & $*$ \\
\hline DB-B vs. DB -EMC & 1 vs. 1 & ns \\
\hline
\end{tabular}

ns: No significativo $(\mathrm{p}>0,05)$. * Diferencia estadística $(\mathrm{p}<0,05){ }^{*}$ Prueba de chi-cuadrado $\mathrm{X}^{2}$. DB: Dieta basal. DB-EMC: Dieta basal +50 $\mathrm{mg} / \mathrm{kg}$ de extracto de Macleaya cordata. DB-B: Dieta basal $+50 \mathrm{~g} / \mathrm{kg}$ de zinc bacitracina.

En este estudio se investigó los efectos beneficiosos de un aditivo natural para el alimento de lechones, derivado de Macleaya cordata sobre los parámetros de crecimiento e incidencia de diarreas, luego del destete, por ser esta etapa la más susceptible a la presentación de disturbios entéricos, y porque la salud del intestino de los lechones es muy importante en la producción porcina exitosa (Sun y Kim, 2017). El EMC contiene un alcaloide denominado sanguinarina y a través del presente estudio se determinó su efecto similar al de la bacitracina, medido en los indicadores de crecimiento y en el control de diarreas, lo cual es concordante con la concepción, que se tiene de los fitobióticos, o productos derivados de plantas, como tal o derivados de las mismas, que al ser ingeridos mejoran el crecimiento y la salud intestinal de los cerdos (Windisch et al., 2008). Por tanto, los extractos vegetales son grandes alternativas alimenticias, al haberse descubierto sus propiedades promotoras de crecimiento y antimicrobianas (Maenner et al., 2014; Zeng et al., 2015). 
Los resultados de este estudio indicaron que la administración del EMC en el alimento en toda la fase experimental post destete tuvo efectos beneficiosos sobre parámetros de crecimiento en lechones, similares al efecto de la bacitracina, en comparación con el grupo control negativo, que no consumió ni EMC ni antibiótico. Este efecto quedó demostrado por el mayor aumento del PC del lechón, mejores GMD e ICA (Tablas 2 y 3). Los resultados actuales están de acuerdo con estudios previos, en los cuales se mejora el rendimiento productivo de cerdos en crecimiento, luego del destete (Kantas et al., 2015). Sin embargo, nuestros resultados están en contraste con el resultado de Blank et al. (2010), que evaluaron cerdos de mayor peso (de 31 a 50 $\mathrm{kg}$ ), utilizando el rendimiento en engorde como criterio para medir la eficacia de la sanguinarina, y no encontraron ningún efecto importante en todos los parámetros de rendimiento, como PC, GMD, IDA e ICA.

Macleaya cordata, es una planta que se encuentra en la lista de componentes aditivos para piensos de animales, registrado por la Autoridad Europea de Seguridad Alimentaria (Franz et al., 2005). Su actividad antimicrobiana, antiinflamatoria e inmunomoduladora del EMC se atribuyen a los alcaloides cuaternarios de benzo fenantridina y protopina, de los que deriva la sanguinarina (Simanek et al., 2003). Estos alcaloides exhiben efectos antimicrobianos contra salmonelosis en pollos de engorde observados en la conversión alimenticia y el índice de eficiencia (Abudabos et al., 2019). La mezcla de alcaloides isoquinolínicos derivados de Macleaya cordata ha demostrado tener potencia antiinflamatoria y promover la productividad de pollos de engorde al protegerlos de enteritis necrótica, cumpliendo su papel como una alternativa a los antibióticos (Xue et al., 2017). El EMC también muestra efectos modificadores de los parámetros de fermentación ruminal y microbiota bacteriana en terneros (Zhang et $a l ., 2019)$. En el presente estudio ha quedado demostrado el efecto eficaz del EMC sobre el control de diarreas en lechones, luego de la primera semana de haber ingerido el alimento con $50 \mathrm{mg}$ de EMC por $\mathrm{kg}$ de dieta.

\section{V.CONCLUSIÓN}

Los resultados de este estudio, evidencian que la ingesta de extracto de Macleaya cordata conteniendo grandes cantidades de alcaloides de sanguinarina en la alimentación de lechones destetados, tiene efectos beneficiosos en su crecimiento y control de la incidencia de diarreas post destete. Por lo que el extracto de Macleaya cordata puede sustituir satisfactoriamente a la bacitracina como promotor de crecimiento y controlador de procesos inflamatorios que devienen en diarreas.

\section{REFERENCIAS BIBLIOGRÁFICAS}

Abudabos, A. M., E. O. S. Hussein, M. H. Ali, y M. Q. Al-Ghadi. 2019. "The effect of some natural alternative to antibiotics on growth and changes in intestinal histology in broiler exposed to Salmonella challenge". Poultry Science 98: 1441-1446.

Blank, R., B. Müller-Siegwardt, y S. Wolffram. 2010. "Sanguinarine does not influence availability or metabolism of tryptophan in pigs." Livestock Science 134: 24-26.

Cadillo, J. 2008. Producción de porcinos. Lima (Perú). Juan Gutemberg.

Casana, C. 2017. El uso de antibióticos en la industria alimentaria y su contribución al desarrollo de resistencias. determinantes de la diseminación de la resistencia a la colistina. Tesis de grado. Universidad de Complutense de Madrid. Madrid(España).

Chen, Y. Z., G. Z. Liu, Y. Shen, B. Chen, y J. G. Zeng. 2009. "Analysis of alkaloids in Macleaya cordata (Willd.) R. Br. using highperformance liquid chromatography with diode array detection and electrospray ionization mass spectrometry". Journal of chromatography A 1216: 2104-2110.

Deng Q., X. Tan, H. Wang, Q. Wang, P. Huang, Y. Li, J. Li, J. Huang, H. Yang, y Y. Yin. 2020. “Changes in cecal morphology, cell proliferation, antioxidant enzyme, volatile fatty acids, lipo- 
polysaccharide, and cytokines in piglets during the postweaning period." Journal of Animal Science 98 (3): skaa046. DOI: 10.1093/jas/skaa046

Díaz, A. 2003. Transformaciones, reactividad y sintesis de protopinas. Tesis de Doctorado. Universidad de Málaga. Málaga (España).

Franz, C., R. Bauer, R. Carle, D. Tedesco, A. Tubaro, y K. Zitterl-Eglseer. 2005. Study on the assessments of plants/herbs, plant/herb extracts and their naturally or synthetically produced components as additives for use in animal production. Parma (Italia): CFT/EFSA/FEEDAP.

Kantas, D., V. G. Papatsiros, P. D. Tassis, L. V. Athanasiou, y E. D. Tzika. 2015. "Effect of a natural feed additive (Macleaya cordata), containing sanguinarine, on the performance and health status of weaning pigs". Animal Science Journal 86: 92-98.

Kosina, P., D. Walterova, J. Ulrichova, V. Lichnovsky, M. Stiborova, H. Rydlova, J. Vicar, V. Krecman, M. J. Brabec, y V. Simanek. 2004. "Sanguinarine and chelerythrine: assessment of safety on pigs in ninety days feeding experiment". Food and Chemical Toxicology 42: 8591.

Li, Y., F. Xu, X.Tong, R. Chen, C. Shen, T. Liang, Q. Chu, y B. Zhou. 2020. "Effects of Macleaya cordata extract on small intestinal morphology and gastrointestinal microbiota diversity of weaned pigs." Livestock Science 237: 104040.

Luridsen, C. 2020. "Effects of dietary fatty acids on gut health and function of pigs pre- and postweaning." Journal of Animal Science 98 (4): skaa086. DOI: $10.1093 / \mathrm{jas} / \mathrm{skaa} 086$

Maenner, K, W. Vahjen, y O Simon. 2014. "Studies on the effects of essential-oil-based feed additives on performance, ileal nutrient digestibility, and selected bacterial groups in the gastrointestinal tract of piglets". J Anim Sci 89:
2106-2112.

Mohammadi Gheisar, M. y I. H. Kim. 2018. "Phytobiotics in poultry and swine nutrition"- a review. Italian Journal of Animal Science 17: 92-99.

Nagai, M. A. y E. K. Buttrey. 2018. Effects of PostWeaning Mat-Feeding Frequency on Piglet Health and Performance. Journal of Animal Science 96 (suppl. 1): 75.

Newton, S. M., C. Lau, S. S. Gurcha, G. S. Besra, y C. W. Wright. 2001. "The evaluation of fortythree plant species for in vitro antimycobacterial activities; isolation of active constituents from Psoralea corylifolia and Sanguinaria Canadensis". Journal of Ethnopharmacology 79: 57-67.

Ni, H., Y. Martínez, G. Guan, R. Rodriguez, D. Mas, H. Peng, M. Valdivie Navarro, y G. Liu. 2016. "Analysis of the Impact of Isoquinoline Alkaloids, Derived from Macleaya cordata Extract, on the Development and Innate Immune Response in Swine and Poultry". BioMed research international 1352146. DOI: 10.1155/2016/1352146

NRC (National Research Council). 1998. Nutrient Requirements of Swine. Washington DC (EEUU): The National Academy Press.

Pellikaan, W. F., N. Andrés-Elias, A. Durand, L. J. G. M. Bongers, S. van Laar-van Schuppen, y D. Torrallardona. "Effect of carob bean gum, spray dried porcine plasma and sanguinarine on fermentation activity in the gut of weanling pigs". Livestock Science 133: 164-168.

Tucureanu, M. M., D. Rebleanu, C. A. Constantinescu, M. Deleanu, G. Voicu, E. Butoi, M. Calin, y I. Manduteanu. 2018. "Lipopolysaccharideinduced inflammation in monocytes/macrophages is blocked by liposomal delivery of Gi -protein inhibitor". International Journal of Nanomedicine 13: 63-76.

Simanek, V., R. Vespalec, A. Sedo, J. Ulrichova, y J. Vicar. 2003. "Quaternary benzo[c]phenant- 
hridine alkaloids - biological activities". En: Chemical Probes in Biology. Schneider, M. P. (ed). Dordrecht (Holanda): Kluwer Academic Publishers,.

Sun, Y. y S.W. Kim. 2017. "Intestinal challenge with enterotoxigenic Escherichia coli in pigs, and nutritional intervention to prevent postweaning diarrhea." Animal Nutrition 3: 322-330.

Waititu S.M., F. Yin, R. Patterson, A. Yitbarek, J.C. Rodriguez-Lecompte, y C. M. Nyachoti. 2017. "Dietary supplementation with a nucleotide-rich yeast extract modulates gut immune response and microflora in weaned pigs in response to a sanitary challenge". Animal 11:2156-2164.

Wang J., B.E. Tan, L. Li, M. Qi, W. Ren, y Y. Yin. 2019. "Dietary glutamine, glutamate, and aspartate supplementation improves morphology and intercellular juntion of small intestine in piglets." Journal animal science 97 (3): 472474.

Windisch, W., K. Schedle, C. Plitzner, y A. Kroismayr. 2008. "Use of phytogenic products as feed additives for swine and poultry." Journal of Animal Science 86: 140-148.

Xu, B.Y., L. Zhao, D. I. Batonon-Alavo, Y. Mercier, D. Qi, y L. Su. 2017. "Increased consumption of methionine by piglets fed with DL-Met or OH-Met strengthens piglets' ability to cope with LPS-induced inflammatory stress during post-weaning period." Journal of Animal Science 95(suppl.4): 351.

Xue, G. D, S. B. Wu, M. Choct, A. Pastor, T. Steiner, y R. A. Swick. 2017. "Impact of a Macleaya cordata-derived alkaloid extract on necrotic enteritis in broilers." Poultry Science 96: 3581-3585.

Zhang, R., W. B. Zhang, Y. L. Bi, Y. Tu, T. Ma, L. F. Dong, H. C. Du, y Q. Y. Diao. 2019. “Sanguinarine and resveratrol affected rumen fermentation parameters and bacterial community in calves". Animal Feed Science and Technology
251:64-75.

Zeng, Z, X. Xu, Q. Zhang, P. Li, P. Zhao, y Q. Li. 2015. "Effects of essential oil supplementation of a low-energy diet on performance, intestinal morphology and microflora, immune properties and antioxidant activities in weaned pigs." Anim SciJ 86: 279-285. 